Supporting Information for

\title{
Electron Holography and Magnetotransport Measurements Reveal Stabilized Magnetic Skyrmions in $\mathrm{Fe}_{1-x} \mathrm{Co}_{x} \mathrm{Si}$ Nanowires
}

\author{
Nitish Mathur ${ }^{\dagger}$, Matthew J. Stolt ${ }^{\dagger}$ Kodai Niitsu ${ }^{\ddagger}$, Xiuzhen $\mathrm{Yu}^{\ddagger}$, Daisuke Shindo ${ }^{\ddagger}$, Yoshinori \\ Tokura ${ }^{\ddagger}$ and Song Jin ${ }^{\dagger *}$ \\ 'Department of Chemistry, University of Wisconsin-Madison, 1101 University Avenue, \\ Madison, Wisconsin, 53706, United States \\ ${ }^{*}$ RIKEN Center for Emergent Matter Science (CEMS), Wako 351-0198, Japan \\ §Institute of Multidisciplinary Research for Advanced Materials, Tohoku University, Sendai 980- \\ 8577, Japan \\ "Department of Applied Physics, University of Tokyo, Tokyo 113-8656, Japan \\ *email: jin@chem.wisc.edu
}




\section{Additional Figures:}

Figure S1: Schematic of chemical vapor deposition synthesis of $\mathrm{Fe}_{1-x} \mathrm{Co} x \mathrm{Si}$ nanowires

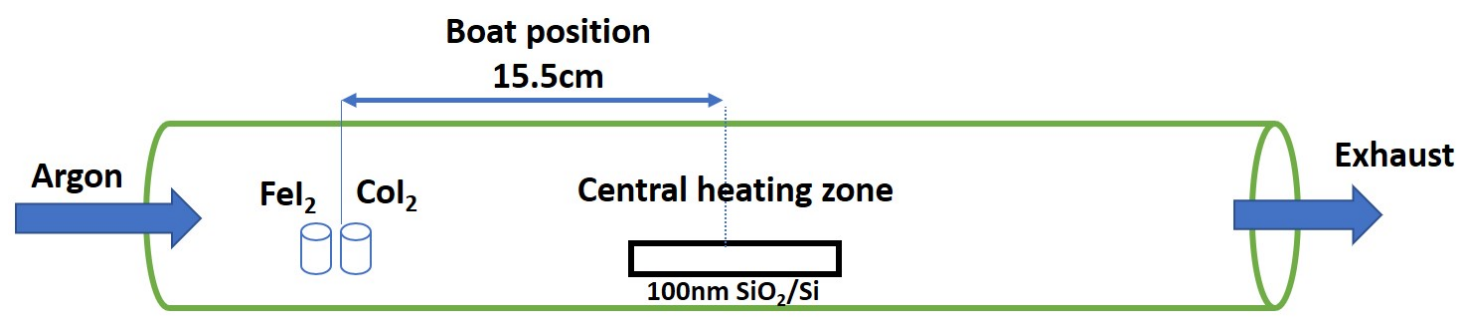

Figure S1. Chemical synthesis of $\mathrm{Fe}_{1-x} \mathrm{Co}_{x} \mathrm{Si}$ alloy nanowires using CVD of two metal iodide precursors. The two precursors were independently vaporized and mixed in gaseous phase and carried downstream in the CVD reactor by argon gas at a flow rate of $190 \mathrm{sccm}$, where decomposition and silicide nanowire formation occur on silicon substrates coated with thin $(\sim 100 \mathrm{~nm})$ silicon oxide placed downstream at $900{ }^{\circ} \mathrm{C}$.

Figure S2: Compositional analysis of $\mathrm{Fe}_{1-x} \mathrm{Co}_{x} \mathrm{Si}$ nanowires on silicon substrate

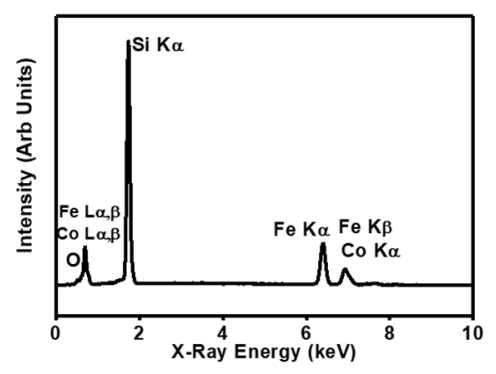

b

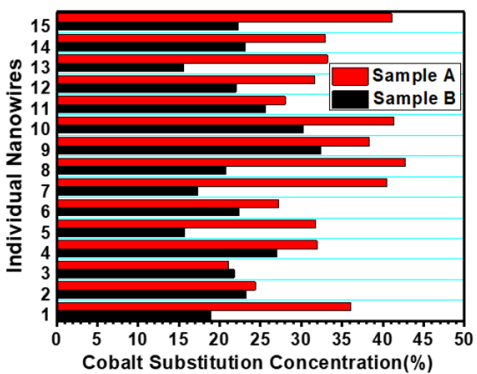

C

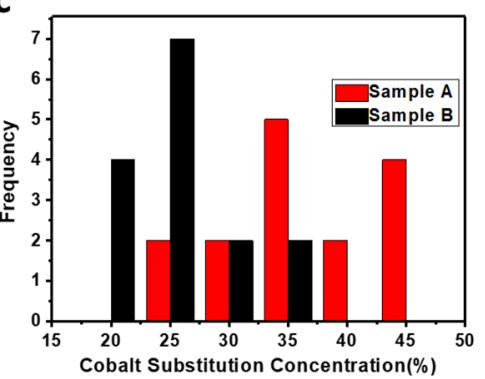

Figure S2. (a) Representative EDS spectrum recorded from the $\mathrm{Fe}_{1-x} \mathrm{Co}_{x} \mathrm{Si}$ NWs. (b) The results of SEMEDS analysis of cobalt substitution concentration (x) represented as histograms (c) for 15 nanowires each from sample A (red bars) and sample B (blue bars). 


\section{Figure S3: Compositional analysis of the NW sample for electron holography}
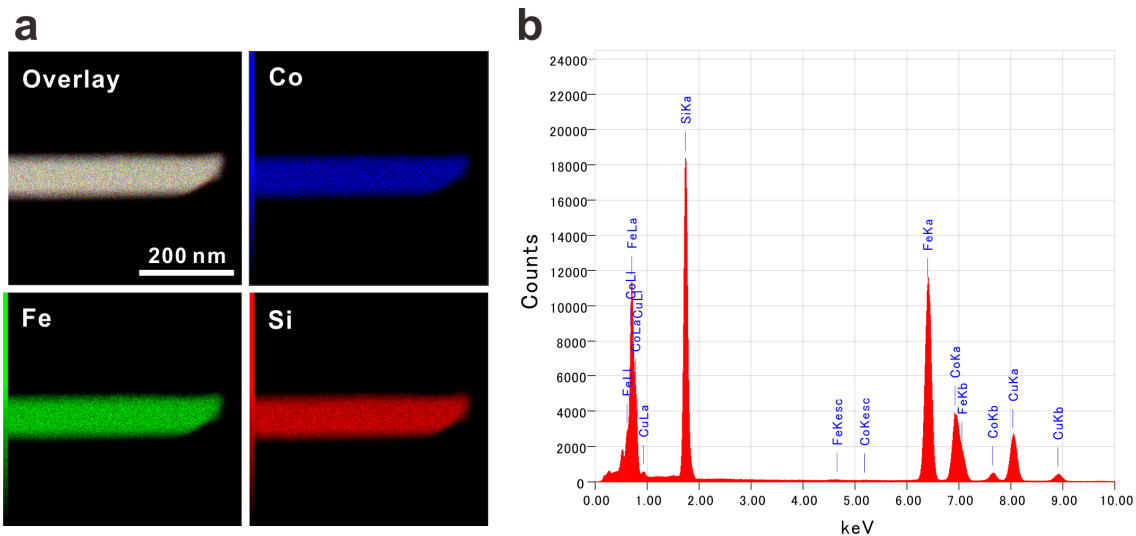

Figure S3. TEM-EDS analysis of the NW studied with off-axis EH observations. (a) TEM-EDS elemental mapping of the NW showing the homogenous elemental distribution. (b) TEM-EDS spectrum of the NW revealing the average composition to be $\mathrm{Fe}_{0.75} \mathrm{Co}_{0.25} \mathrm{Si}$.

Surface and cross-sectional geometry of the NW sample for electron holography: Above $T_{\mathrm{C}}$, the phase shift is brought about by the mean inner potential of materials, which is basically responsible for the traversing thickness. Figure $\mathrm{S} 4$ shows reconstructed phase images at $T=60 \mathrm{~K}$ and $H=0$ Oe in the forms of (a) $\cos (20 \varphi)$ and (b) color-coded $\nabla \varphi$. These images reveal that the NW consists of flat facets forming a rhombohedral cross-section similarly to the NWs shown in the inset of Figure 1a. In addition, we can see phase gradation in vacuum with a summit near the tip of the NW. This is presumably caused by electrical charging due to poor electrical conductivity
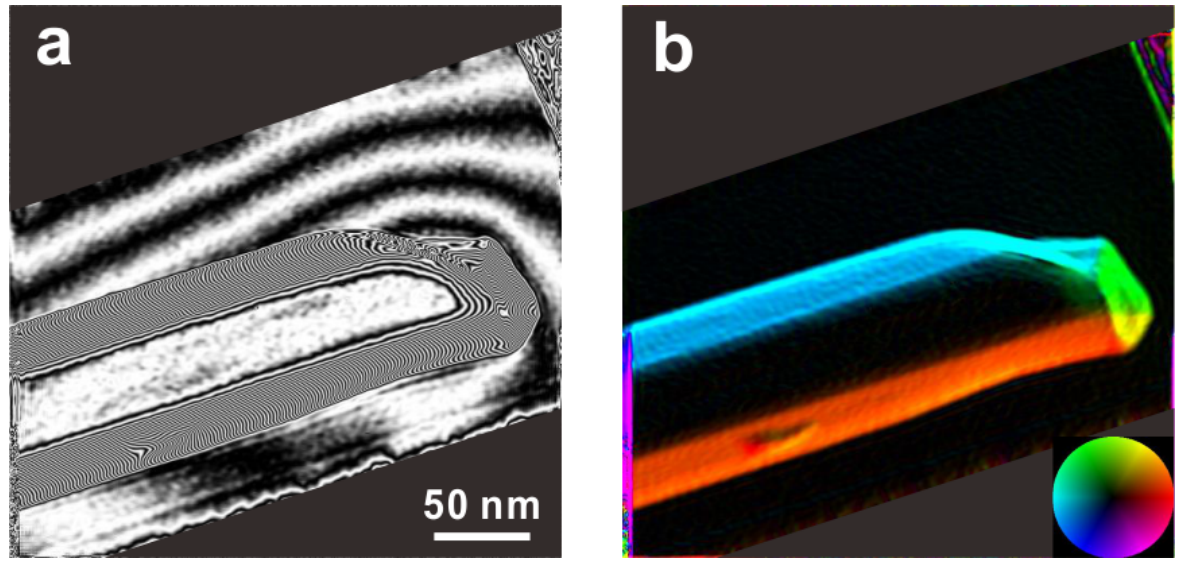

Figure S4. Surface and cross-sectional geometry of the NW sample for electron holography. Reconstructed phase images at $T=60 \mathrm{~K}$ and $H=0$ Oe in the forms of (a) $\cos (20 \varphi)$ and (b) color-coded $\nabla \varphi$ where the color hue and brightness represent $\operatorname{atan} 2(\nabla \varphi)$ and $|\nabla \varphi|$, respectively. 
around the tip. We suspect that the rugged shape of the tip was formed by cleavage fracture. Introduced nano-sized cracks may also depress the surrounding electrical conductivity.

\section{Figure S5: Optical conditions of electron holography observations}
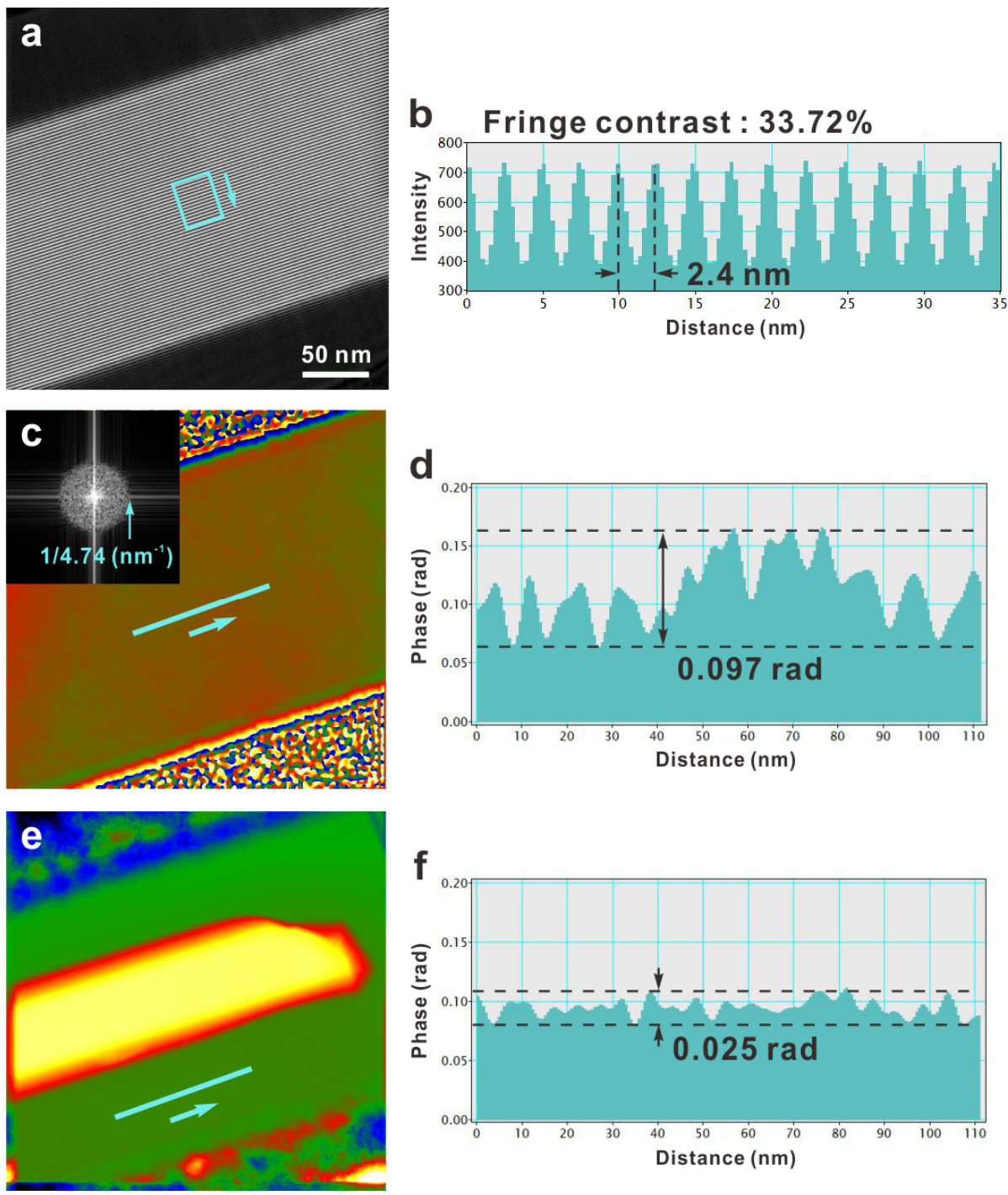

Figure S5. Optical conditions of electron holography observations. (a) Representative hologram. (b) Intensity profile of the blue rectangle area marked in (a). (c) Reconstructed phase image of (a). Inset shows Fourier transform pattern. (d) Phase profile of the blue line drawn in (c). (e) Phase image at $T=$ $10 \mathrm{~K}$ and $H=0 \mathrm{Oe}$, wherein 100 reconstructed ones are averaged. (f) Phase profile of the blue line drawn in (e). 
Image processing of reconstructed phase images for reducing unwanted artefacts: Figure S6a shows the residual phase image at $T=10 \mathrm{~K}$ and $H=0$ Oe after subtraction by the phase image taken at $60 \mathrm{~K}$ and 0 Oe. Broad bands inside the NW running along the long axis are the undersubtracted/oversubtracted $\varphi$ MIP components, which are caused by slight geometric misalignment during temperature change. Besides, fine stripes are visible even in vacuum. These stripes are introduced by the Fresnel diffraction at the lower biprism. The Fresnel fringes should be sufficiently suppressed in the double-biprism interferometry ${ }^{\mathrm{S} 1}$ but become apparent if the lower biprism is away from the shade of the upper one. The amplitude of these artefacts is $\sim 0.1 \mathrm{rad}$, which is less than $1 \%$ of the maximum $\varphi_{\text {MIP }}(\sim 11.3 \mathrm{rad})$ but is still comparable to the $\varphi_{\mathrm{MAG}}$ of helices and skyrmions. To remove these unwanted artefacts, we employed additional process which subtracts the phase components that are unchanged along the NW direction. The processed images (Figure S6b) can capture the helical stripes more clearly. Finally, the second-order Butterworth filtering ${ }^{\mathrm{S} 2}$ was employed to smooth short-range phase undulations and result in Figure S6c.
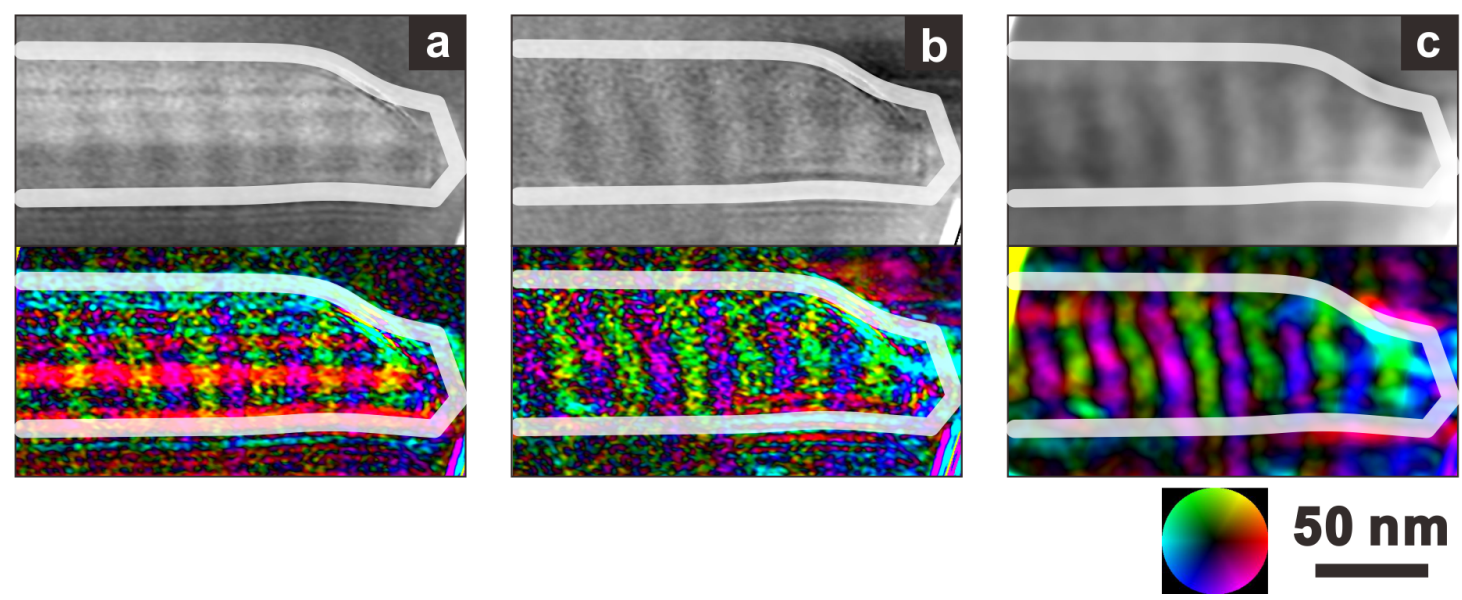

Figure S6. Image processing of reconstructed phase images for reducing unwanted artefacts. (a) Reconstructed phase image at $T=10 \mathrm{~K}$ and $H=0$ Oe after subtraction by that at $T=60 \mathrm{~K}$ and $H=0$ Oe. (b) Phase image of (a) after further subtraction by the unchanged components along the NW long axis. (c) Phase image of (b) after the second-order Butterworth filtering. The upper panels are the grayscale phase $(\varphi)$ maps and the lower panels display in the form of $\nabla \varphi$ where the color hue and brightness correspond to $\operatorname{atan} 2(\nabla \varphi)$ and $|\nabla \varphi|$, respectively. 
Four probe $\mathrm{NW}$ device fabrication for magnetoresistance measurements: The $\mathrm{Fe}_{1-x} \mathrm{Co}_{x} \mathrm{Si}$ NWs from Sample A and B were transferred to Si (100) substrate with a $600 \mathrm{~nm}$ silicon oxide coating prior to device fabrication. NWs were dispersed in isopropanol (IPA) solution via ultrasonication and drop casted on the substrates. Photoresist layers of MMA and PMMA was spin coated onto the substrates and the intended areas of PMMA resist were exposed with e-beam lithography using a nano-pattern generation system (NPGS) to fabricate metal electrodes, then the defined patterns were developed in a MIBK (Methyl isobutyl ketone) /IPA developer solution for $30 \mathrm{~s}$. After etching with buffered HF solution for $15 \mathrm{~s}$ to remove the silicon oxide layer on the surface of the NW, non-magnetic metallic contacts were fabricated for NW electrodes $(\sim 1 \mu \mathrm{m}$ in width) as a combination of Ti layer covered with Au using e-beam deposition. Importantly, a twostep electrode deposition process was implemented to achieve the most consistent device contact and low $\mathrm{S} / \mathrm{N}$ ratios. In the first evaporation, the substrate was set perpendicular to the evaporation direction and $50 \mathrm{~nm}$ layers of both $\mathrm{Ti}$ and $\mathrm{Au}$ were evaporated. The second step involves orienting
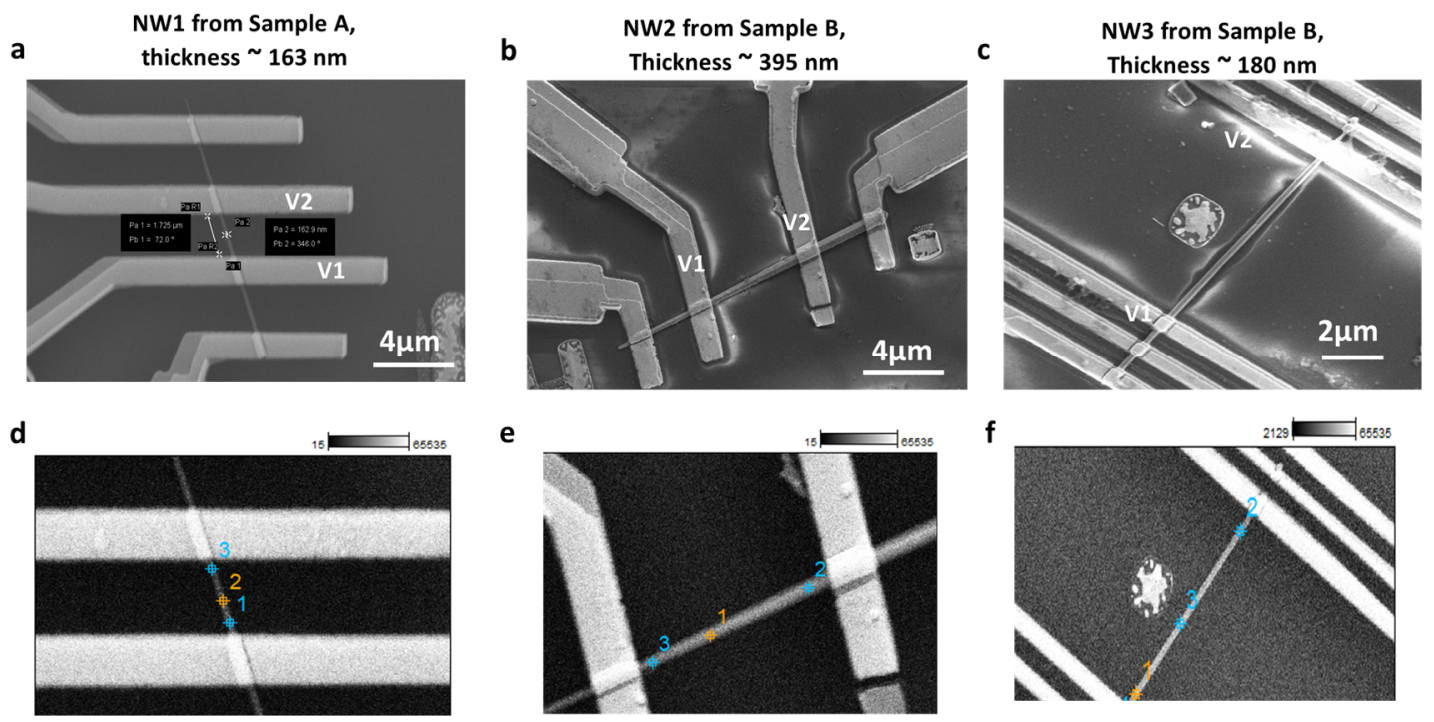

Figure S7. The SEM images of four probe device on NW1(a), NW2(b) and NW3(c). V1 and V2 represents voltage leads used for measurement of magnetoresistance. Dimensions were measured from SEM images as shown in (a). (d-f) Magnified images of probing area between the voltage leads of four probe devices on NW 1-3 respectively for SEM-EDS compositional analysis. 
the substrate on a custom-built stage attachment so that the substrate will be angled $45^{\circ}$ with respect to the evaporation direction to deposit $70 \mathrm{~nm}$ of $\mathrm{Ti}$ and $50 \mathrm{~nm}$ of $\mathrm{Au}$. The angled evaporation steps allow for better side wall metal contact to the NWs resulting in better $\mathrm{S} / \mathrm{N}$ devices. The SEM images of the three NW devices measured are shown in Figure S5.

Table S1: The average Co\% $(x)$ is determined via SEM-EDS conducted at 3 spots between the voltage leads of the fabricated devices for all 3 NWs (Figure S7d-f).

\begin{tabular}{|c|c|c|c|}
\hline & Atomic\% Fe_K & Atomic\% Co_K & Co substitution \\
\hline NW1_Spot 1 & 3.74 & 2.22 & 0.37 \\
\hline NW1_Spot 2 & 3.35 & 2.26 & 0.40 \\
\hline NW1_Spot 3 & 3.86 & 2.09 & 0.35 \\
\hline & $\begin{array}{c}\text { Average Co } \\
\text { substitution }(x)\end{array}$ & $\begin{array}{c}\text { Standard } \\
\text { Deviation in } x\end{array}$ & $\begin{array}{c}\text { Standard error in } \\
\text { x }\end{array}$ \\
\hline & 0.37 & 0.02 & 0.01 \\
\hline
\end{tabular}

\begin{tabular}{|c|c|c|c|}
\hline & Atomic\% Fe_K & Atomic\% Co_K & Co substitution \\
\hline NW2_Spot 1 & 7.62 & 1.82 & 0.19 \\
\hline NW2_Spot 2 & 9.75 & 2.12 & 0.18 \\
\hline NW2_Spot 3 & 1.30 & 0.48 & 0.27 \\
\hline & $\begin{array}{c}\text { Average Co } \\
\text { substitution (x) }\end{array}$ & $\begin{array}{c}\text { Standard } \\
\text { Deviation in } \mathrm{x}\end{array}$ & $\begin{array}{c}\text { Standard error in } \\
\mathrm{x}\end{array}$ \\
\hline & 0.21 & 0.04 & 0.02 \\
\hline
\end{tabular}

\begin{tabular}{|c|c|c|c|}
\hline & Atomic\% Fe_K & Atomic\% Co_K & Co substitution \\
\hline NW3_Spot 1 & 6.02 & 1.24 & 0.17 \\
\hline NW3_Spot 2 & 5.85 & 1.64 & 0.22 \\
\hline NW3_Spot 3 & 6.57 & 1.31 & 0.17 \\
\hline & $\begin{array}{c}\text { Average Co } \\
\text { substitution(x) }\end{array}$ & $\begin{array}{c}\text { Standard } \\
\text { Deviation in } x\end{array}$ & $\begin{array}{c}\text { Standard error in } \\
\text { x }\end{array}$ \\
\hline & 0.19 & 0.02 & 0.01 \\
\hline
\end{tabular}


Figure S8: Temperature dependent MR for NW1 and NW2 under $H_{\perp}$
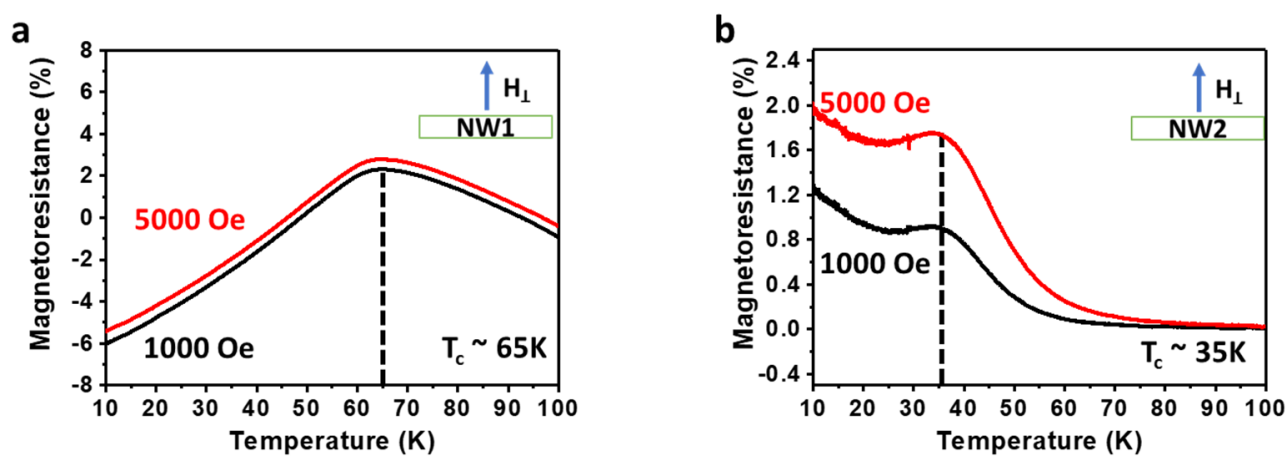

Figure S8. Temperature dependent MR of (a) NW1 and (b) NW2 with perpendicular applied magnetic field, $\mathrm{H}_{\perp}$.

Figure S9: MR-H isotherms between $10-32 \mathrm{~K}$ under $\mathrm{H}_{\perp}$ for the $\mathrm{Fe}_{0.81} \mathrm{Co}_{0.19} \mathrm{Si} \mathrm{NW}$ (NW3)

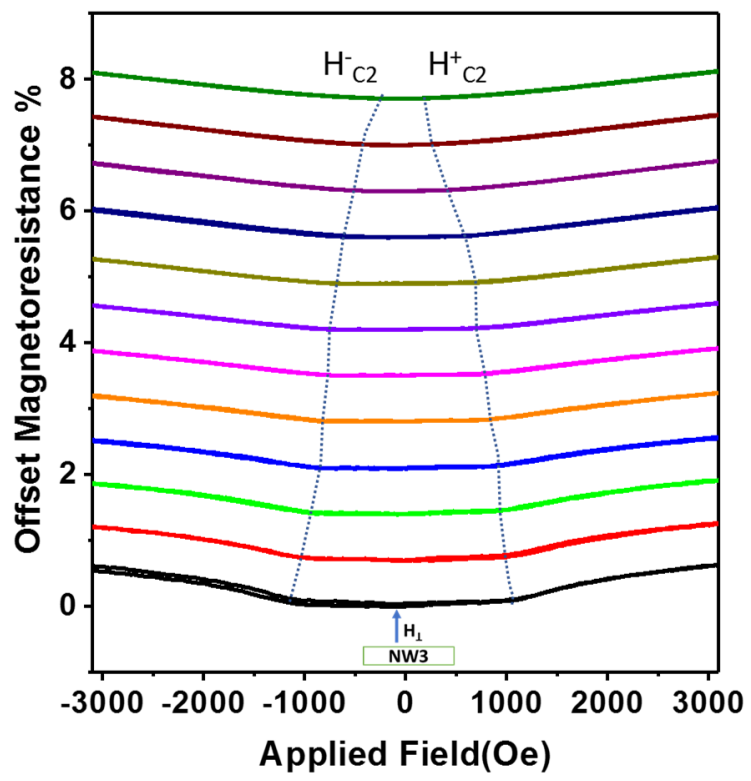

Figure S9. Offset MR-H isotherms between 10-32K under $\mathrm{H}_{\perp}$ for the $\mathrm{Fe}_{0.81} \mathrm{Co}_{0.19} \mathrm{Si} \mathrm{NW}(\mathrm{NW} 3) . \mathrm{H}_{\mathrm{C} 2}$ represents critical field for skyrmion to field polarized spin state transition marked by the dotted blue lines on MR-H isotherms. 


\section{References:}

S1. Harada, K.; Tonomura, A.; Togawa, Y.; Akashi, T.; Matsuda, T., Double-Biprism Electron Interferometry. Appl. Phys. Lett. 2004, 84, 3229-3231

S2. Voelk1, E.; Allard, L. F.; Frost, B., Electron Holography: Recent Developments. Scanning Microsc. 1997, 11, 407-416 